\title{
La moda en su laberinto: Mass media, moda e hipermodernidad Proyecto de Investigación $\mathrm{N}^{\circ} 17.3$ \\ Equipo de Investigación ${ }^{(1)}$ \\ Patricia Doria
}

por Facultad de Diseño y Comunicación, Universidad de Palermo (ARG)

\begin{abstract}
Resumen: El Proyecto de Investigación 17.3 avanza sobre un análisis que toma su punto de partida en la observación de los interrogantes y respuestas de la Moda en la hipermodernidad. Como el Hilo de Ariadna, se introduce por los vericuetos de la investigación en moda cuyos relatos simultáneos y segmentados van construyendo las miradas válidas sobre el objeto, y donde el recorrido resulta ser más importante que el objetivo. Se analiza el sistema de la moda, desde sus múltiples aristas en un complejo y exhaustivo recorrido multidisciplinario, un armado del sustrato vivo, la base y el sostén de un laberinto plural y multidireccional de análisis, en el que discurren tres miradas: una orientada particularmente a las variables de relación entre moda y sociedad, otra donde el eje principal de abordaje discurre en el contrapunto moda y cultura, y por último, un tercero que se orienta hacia la innovación a través del análisis de casos.
\end{abstract}

Palabras clave: Moda - Sociedad - Innovación - Cultura - Diseño - Investigación

[Resúmenes en inglés y portugués en la página 269]

${ }^{(1)}$ Los CVs del Equipo de Investigación pueden consultarse en el Capítulo Directores de Líneas y Coordinadores de Proyectos de esta misma Edición.

\section{Acerca del Proyecto 17.3}

\section{La moda en su laberinto:}

\section{Mass media, moda e hipermodernidad}

El Proyecto 17.3 La moda en su laberinto: Mass media, moda e hipermodernidad, analiza el sistema de la moda desde la recombinacion de elementos existentes, la representación del poder individual, la libertad de lo plural, los discursos múltiples e inter-dimensionales, que habilitados como relatos simultáneos y segmentados van construyendo distintos en- 
foques sobre el objeto moda, de acuerdo a tres miradas: Moda y Sociedad, Moda y Cultura y Moda e Innovación. Pretende impactar en la actualización de los contenidos dictados en las carreras de grado y posgrado, pudiendo contribuir con la capacitación profesional de los diseñadores para una reflexión sobre su propia práctica.

Avanza en una investigación en la que participan académicos de distintas Instituciones, con la coordinación de Patricia Doria (UP), con el fin de generar un corpus sólido de conocimientos sobre los diversos escenarios socioculturales en los que se desarrolla la moda en relación a los medios de comunicación de masas y las condiciones de la hipermodernidad, a través de la articulación de producciones teóricas de profesionales de la moda, la imagen y las tendencias convocados a reflexionar sobre las diversas dimensiones del campo en estudio.

Las reflexiones y los resultados obtenidos en el Proyecto 17.3 La Moda en su Laberinto, son continuación del Proyecto 17.1 Cine, Moda, Cuerpo, Arte y Diseño, y guardan relación con el 17.2 Comunicación e Imagen personal $360^{\circ}$ y $17.4 \mathrm{La}$ Imagen personal frente a los nuevos desafíos 2020, en el marco de la misma Línea de Investigación y bajo la misma Directora Patricia Doria.

Sus principales objetivos son:

- Analizar las distintas variables del sistema de la moda en la hipermodernidad centrados en las intersecciones de la Moda y los constructos sociales

- Problematizar y teorizar acerca de los discursos del sistema de la moda, en tanto sociedad, cultura, historia y épocas en sus espacios múltiples e inter-dimensionales - Estudiar como los elementos existentes y los nuevos se van conjugando y recombinando de un período a otro, de un diseñador a otro, de un massmedia a otro, y cómo juega un papel fundamental la creación y la innovación

\section{Acerca de la Línea 17}

Imagen, Moda y Tendencias dirigida por Patricia Doria se desarrolla de manera ininterrumpida desde 2015 en la Facultad de Diseño y Comunicación (UP, Argentina), e incluye hasta el momento cuatro proyectos finalizados el 17.1 Cine, Moda, Cuerpo, Arte y Diseño coordinado por Patricia Doria (UP), Denise Trindade (Universidade Estácio de Sá, Brasil) y Beatriz Ferreira Pires (Escola de Artes, Ciências e Humanidades, Universidade de São Paulo EACH-USP, Brasil), el 17.2 Comunicación e Imagen personal $360^{\circ}$ coordinado por María Pia Estebecorena y Susy Inés Bello Knoll (UP y AICI), el 17.3 La Moda en su Laberinto coordinado por Patricia Doria (UP), y el 17.4 La Imagen personal frente a los nuevos desafíos 2020 coordinado por María Pía Estebecorena (UP y AICI). 


\section{Mapa de Áreas y Proyectos}

El Proyecto 17.3 se vincula con todas las carreras de grado y posgrado correspondientes a la Facultad de Diseño y Comunicación, y está claramente enlazado a las carreras de indumentaria, moda e imagen: Comunicación de Moda, Diseño de Moda, Fotografía de Moda, Marketing de la Moda, Moldería \& Confección y Producción de Moda. Guarda relación con la serie de proyectos de la Línea 12. Nuevos paradigmas en la enseñanza de la moda y el diseño y en la Línea 5. Cuerpo y Vestuario.

\section{Productos y Resultados}

\section{a)- Publicaciones}

Cuaderno del Centro de Estudios de Diseño y Comunicación No100. (2021) La Moda en su Laberinto. Coordinación Patricia Doria. Facultad de Diseño y Comunicación Año XX, Diciembre 2019, Buenos Aires, Argentina. ISSN: 1668-0227. Esta publicación documenta y comunica los resultados alcanzados en el proyecto de investigación 17.3 La Moda en su Laberinto. Mass media, Moda e Hipermodernidad, y a continuación se detallan los autores $y$ artículos contenidos en ella:

Doria, Patricia M. (2021) Introducción. Interrogantes y respuestas de la Moda en la hipermodernidad (Pp. 11 a 15)

Lopez Rizzo, María Belén (2021) Masculinidad y Moda: el Dandismo en Argentina (Pp. 17 a 26)

Tidele, Jesica (2021) Moda y feminismo: la vestimenta como símbolo de protesta (Pp. 27 a 40)

Tuozzo, María Valeria (2021) Hipermoda, la moda rizoma (Pp. 41 a 50)

Moreira Bravo, Yamila L (2021) La simbología del traje sastre femenino y el discurso de emancipación femenina (Pp. 51 a 65)

Castro, Jorge (2021) La Industria Textil y de la Moda, Responsabilidad Social y la Agenda 2030 (Pp. 67 a 85)

Peisajovich, Sara (2021) Desfile de moda: arte y performance (Pp. 85 a 95)

Pérez, Lorena (2021) Observar y consumir moda. Nuevas formas de comunicación digital (Pp. 97 a 107)

Gómez del Rio, Gabriela (2021) Los cibergéneros especializados: análisis sobre la modalidad de gestión de contenidos en weblogs independientes de moda (Pp. 109 a 125)

Galletti, Patricia Cecilia (2021) Prolijidad y corrección. Vectores de normalización y socialización interclase para el cuidado del cliente de elite en una marca comercial porteña (Pp. 127 a 137)

Gómez García, Cecilia (2021) Conservación preventiva de colecciones de vestuario escénico. Colección de vestuario compañía de danza española Ángel Pericet (Pp. 139 a 154)

Turnes, Cecilia (2021) Moda y Vestuario: universos paralelos con infinitas posibilidades de encuentro (Pp. 155 a 165) 
Insausti, Florencia (2021) Moda, publicidad y derecho (Pp. 167 a 178)

Echeverría, Pamela (2021) Proteger las creaciones en el mundo de la moda (Pp. 179 a 187)

Rudi, Constanza Soledad (2021) Emprender en el Mundo de la Moda (Pp. 189 a 199)

Spina, María Laura (2021) La nueva trama de Burberry (Pp. 201 a 215)

Scalisse, Valeria (2021) Transgresión y glamour, las portadas de la moda. Un análisis de la pasarela/ vidriera de papel (Pp. 217 a 230)

Tesoriere, Pablo Andrés (2021) Fashion film: tendencia mundial en comunicación (Pp. 231 a 240)

Moscoso Barcia, Yanina M. (2021) Cosmovisión textil actual (Pp. 241 a 252)

Mihanovich, María (2021) Slow fashion en tiempo de redes sociales (Pp. 253 a 264)

Medina Matteazzi, Paola (2021) Tecnología 3D en el calzado. Artesanato y tradición (Pp. 265 a 312)

\section{b)- Congresos / Coloquios / Plenarios}

V Coloquio de Investigación y Desarrollo en Diseño Latino. Universidad de Palermo, 27 de julio de 2020. XI Congreso Latinoamericano de Enseñanza del Diseño. Semana Internacional del Diseño en Palermo.

Se presentaron los resultados y avances de la Línea de Investigación №17: Imagen, Moda y Tendencias, en dos comisiones Proyecciones de la Moda y La Imagen Personal frente a los nuevos desafíos 2020 .

En la comisión Proyecciones de la Moda, se presentaron las reflexiones y conclusiones del proyecto 17.3 La Moda en su Laberinto (Finalizado) expusieron: María Belén Lopez Rizzo, Jesica Tidele, María Valeria Tuozzo, Yamila L. Moreira Bravo, Jorge Castro, Sara Peisajovich, Lorena Pérez, Gabriela Gómez del Rio, Patricia Cecilia Galletti, Cecilia Gómez García, Cecilia Turnes, Florencia Insausti, Pamela Echeverría, Constanza Soledad Rudi, María Laura Spina, Valeria Scalisse, Pablo Andrés Tesoriere, Yanina Moscoso Barcia, María Mihanovich, Paola Medina Matteazzi, Patrizia Calefato, Valeria Scalise, Patricia Cecilia Galletti, Diego Labrin Ladrón de Guevara, Claudia Herrera Ramos, Mónica Incorvaia, Pamela Echeverría, Martina Luski, Helga Mariel Soto, María Cecilia Guarás, Zineb El Habchi Mahir, Graciela Padilla Castillo, Pablo Tesoriere, Paula Negro, Yanina Moscoso Barcia, Patricia Marina Doria, Eugenia Bailo Donnet, Sara Peisajovich, María Cecilia Turnes y Paola Matteazzi.

A continuación se detallan las ponencias presentadas en esta Comisión coordinada por la Directora del proyecto Patricia Doria:

María Belén Lopez Rizzo (Argentina)

Masculinidad y Moda: el Dandismo en Argentina

Jesica Tidele (Argentina)

Moda y feminismo: la vestimenta como símbolo de protesta 
María Valeria Tuozzo (Argentina)

Hipermoda, la moda rizoma

Yamila L. Moreira Bravo (Argentina)

La simbología del traje sastre femenino y el discurso de emancipación femenina Jorge Castro (Uruguay)

La Industria Textil y de la Moda, Responsabilidad Social y la Agenda 2030

Sara Peisajovich (Argentina)

Desfile de moda: arte y performance

Lorena Pérez (Argentina)

Observar y consumir moda. Nuevas formas de comunicación digital

Gabriela Gómez del Rio (Argentina)

Los cibergéneros especializados: análisis sobre la modalidad de gestión de contenidos en weblogs independientes de moda

Patricia Cecilia Galletti (Argentina)

Prolijidad y corrección. Vectores de normalización y socialización interclase para el cuidado del cliente de elite en una marca comercial porteña

Cecilia Gómez García (Argentina)

Conservación preventiva de colecciones de vestuario escénico. Colección de vestuario compañía de danza española Ángel Pericet

Cecilia Turnes (Argentina)

Moda y Vestuario: universos paralelos con infinitas posibilidades de encuentro

Florencia Insausti (Argentina)

Moda, publicidad y derecho

Pamela Echeverría (Argentina)

Proteger las creaciones en el mundo de la moda

Constanza Soledad Rudi (Argentina)

Emprender en el Mundo de la Moda

María Laura Spina (Argentina)

La nueva trama de Burberry

Valeria Scalisse (Argentina)

Transgresión y glamour, las portadas de la moda. Un análisis de la pasarela/ vidriera de papel

Pablo Andrés Tesoriere (Argentina)

Fashion film: tendencia mundial en comunicación

Yanina Moscoso Barcia (Argentina)

Cosmovisión textil actual

María Mihanovich (Argentina)

Slow fashion en tiempo de redes sociales

Paola Medina Matteazzi (Argentina)

Tecnología 3D en el calzado. Artesanado y tradición

Patrizia Calefato (Italia)

Fashionscapes, La moda nel mondo iperconneso 
Valeria Scalise (Argentina)

Un siglo de moda y alta costura oficial, que trascendió París. L'Officiel de la couture et de la mode de París

Patricia Cecilia Galletti (Argentina)

Pedagogía, Diseño y Alteridad: una experiencia áulica con jóvenes gitanas en situación de vulnerabilidad

Diego Labrin Ladrón de Guevara (Perú)

Investigación creativa en la era del hipervínculo

Claudia Herrera Ramos (Perú)

El poder del textil en los constructos sociales identitarios

Mónica Incorvaia (Argentina)

Fotografía y moda. Aliados indispensables

Pamela Echeverría (Argentina)

Fashion Law, La tecnología al servicio de la moda

Martina Luski (Argentina)

Moda Inteligente

Helga Mariel Soto (Argentina)

Nuevas encrucijadas en la moda masculina

María Cecilia Guarás

Contracultura, moda, ética y estética: un recorrido cultural intra sistémico

Zineb El Habchi Mahir y Graciela Padilla Castillo (España)

Futbolistas de élite como instagramers de moda: Ronaldo y Messi

Pablo Tesoriere (Argentina)

Comunicación visual en redes

Paula Negro (Argentina)

La Moda en tiempos de Pandemia

Yanina Moscoso Barcia (Argentina)

El rastro de la moda

Patricia Marina Doria y Eugenia Bailo Donnet (Argentina)

La ley de la atracción. Mensajes Ocultos, la simbología como nuevo lenguaje identitario de las marcas de moda

Sara Peisajovich (Argentina)

El arte visual en las tapas de revistas Vogue y Harper's Bazaar de 1920 a 1950

María Cecilia Turnes (Argentina)

Un cuerpo que viste: breve estudio sobre la prenda y su capacidad transformadora

Paola Matteazzi (Argentina)

El calzado en el cine

$3^{\circ}$ Plenario de Directores de Investigación DC. 26, 27 y 29 de agosto de 2019. En este tercer plenario los Directores de las Líneas y Proyectos de Investigación presentaron a sus pares y al conjunto del Programa de Investigación de la Facultad de Diseño y Comunicación, los resultados obtenidos y/o en proceso (publicaciones y acuerdos), junto con los avances de Proyectos. La Directora de la Línea de Investigación №17 presentó a sus pares, 
el debate y los resultados del Proyecto 17.3 La Moda en su Laberinto y el Proyecto 17.4 La Imagen Personal frente a los nuevos desafíos 2020.

\title{
c)- Formación de Posgrado e Impacto curricular
}

La Directora Patricia Doria forma parte del Cuerpo Académico de la Facultad de Diseño y Comunicación e incorpora los contenidos se su investigación a sus asignaturas.

\begin{abstract}
Research Project 17.3 advances on an analysis that takes its starting point in the observation of the questions and answers of Fashion in hypermodernity. Like the Thread of Ariadna, it is introduced through the twists and turns of fashion research whose simultaneous and segmented stories build valid views on the object, and where the route turns out to be more important than the objective. The fashion system is analyzed from its multiple edges in a complex and exhaustive multidisciplinary journey, an assembly of the living substrate, the base and the support of a plural and multidirectional labyrinth of analysis, in which three views run: one particularly oriented to the relationship variables between fashion and society, another where the main axis of approach runs in the fashion and culture counterpoint, and finally, a third that is oriented towards innovation through the analysis of cases.
\end{abstract}

Keywords: Fashion - Society - Innovation - Culture - Design - Research

Resumo: O Projeto de Pesquisa 17.3 avança em uma análise que parte da observação das perguntas e respostas da Moda na hipermodernidade. Como o Fio de Ariadna, é introduzido por meio das voltas e reviravoltas da pesquisa de moda cujas histórias simultâneas e segmentadas constroem visões válidas sobre o objeto, e onde o percurso acaba sendo mais importante do que o objetivo. O sistema da moda é analisado a partir de suas múltiplas arestas em uma jornada multidisciplinar complexa e exaustiva, uma montagem do substrato vivo, a base e o suporte de um labirinto de análise plural e multidirecional, no qual correm três visões: uma particularmente orientada às variáveis de relacionamento entre moda e sociedade, outra onde o eixo principal de abordagem corre no contraponto da moda e da cultura e, por fim, uma terceira que se orienta para a inovação através da análise de casos.

Palavras chave: Moda - Sociedade - Inovação - Cultura - Design - Pesquisa

[Las traducciones de los resúmenes fueron realizadas a través de traductor automático] 